\title{
Injeção Intravascular Acidental de Ropivacaína a 0,5\% durante a Realização de Anestesia Peridural Torácica. Relato de Casos *
}

\author{
Accidental Intravascular Injection of $0.5 \%$ Ropivacaine \\ during Thoracic Epidural Anesthesia. Case Reports \\ Fábio Geraldo Curtis, TSA ${ }^{1}$; Robson Furlani ${ }^{2}$; Yara Marcondes Machado Castiglia, TSA ${ }^{3}$
}

\section{RESUMO}

Curtis FG, Furlani R, Castiglia YMM - Injeção Intravascular Acidental de Ropivacaína a 0,5\% durante a Realização de Anestesia Peridural Torácica. Relato de Casos

JUSTIFICATIVA E OBJETIVOS: A ropivacaína foi introduzida na prática clínica há pouco mais de dez anos, associando-se a baixo risco de complicações do sistema nervoso central e cardiovascular. O objetivo destes relatos é apresentar um caso de parada cardíaca e outro de toxicidade neurológica, após injeção intravascular acidental da ropivacaína, durante a realização de anestesias peridurais.

RELATO DOS CASOS: Trata-se de duas pacientes submetidas a cirurgias plásticas estéticas sob anestesia peridural torácica com ropivacaína a 0,5\%. Durante a realização da técnica, uma delas apresentou parada cardíaca em assistolia e a outra, toxicidade neurológica. Prontamente atendidas, ambas apresentaram rápida recuperação, tendo sido possível a realização dos respectivos atos cirúrgicos.

CONCLUSÕES: O reconhecimento e o tratamento rápidos da injeção intravascular acidental, bem como as características farmacológicas da ropivacaína foram decisivos, em ambos os casos, na boa recuperação das pacientes.

Unitermos: ANESTÉSICOS, Local: ropivacaína; COMPLICAÇÕES: injeção acidental; TÉCNICAS ANESTÉSICAS, Regional: peridural

\section{SUMMARY}

Curtis FG, Furlani R, Castiglia YMM - Accidental Intravascular Injection of $0.5 \%$ Ropivacaine during Thoracic Epidural Anesthesia. Case Reports

BACKGROUND AND OBJECTIVES: Ropivacaine was introduced in the clinical practice a little more than 10 years ago, and has been associated to low risk for central nervous system and cardiovascular complications. These reports aimed at presenting a case of cardiac arrest and another one of neurological toxicity after accidental intravascular ropivacaine injection during epidural anesthesia.

CASE REPORTS: Two patients undergoing cosmetic plastic surgeries were submitted to thoracic epidural anesthesia with $0.5 \%$ ropivacaine. After anesthetic injection, one has presented cardiac arrest in asystole and the other had signs of neurological toxicity. Patients were promptly treated and presented fast recovery, in a way that both surgical procedures could be performed.

CONCLUSIONS: The prompt Identification and treatment of the accidental intravascular injection, as well as ropivacaine pharmacological profile, were decisive in both cases for the satisfactory recovery of our patients.

Key Words: ANESTHETICS, Local: ropivacaine; ANESTHETIC TECHNIQUES, Regional: epidural; COMPLICATIONS: accidental injection

\section{INTRODUÇÃO}

Asive rop ropivacaína foi introduzida na prática clínica como possivel alternativa mais segura à bupivacaína ${ }^{1}$. É bastante utilizada atualmente em anestesias regionais justamente por suas características peculiares - menor neuro e cardio-

\footnotetext{
* Recebido do (Received from) Departamento de Anestesiologia da Faculdade de Medicina de Botucatu - FMB - UNESP, Botucatu, SP

1. Mestre em Anestesiologia/Membro do CET/SBA do Departamento de Anestesiologia da FMB-UNESP; Anestesiologista do Hospital Santa Helena e da Clínica Pardo Oftalmologia de São José do Rio Preto, SP

2. Anestesiologista do Hospital Santa Helena e da Clínica Pardo Oftalmologia de São José do Rio Preto, SP

3. Professora Titular do Departamento de Anestesiologia da FMB-UNESP
}

Apresentado (Submitted) em 17 de março de 2004

Aceito (Accepted) para publicação em 21 de julho de 2004

Endereço para correspondência (Correspondence to)

Dr. Fábio Geraldo Curtis

Rua Coronel Spínola de Castro, 4061/83

15015-500 São José do Rio Preto, SP

E-mail: fabio.curtis@uol.com.br

(C) Sociedade Brasileira de Anestesiologia, 2004 toxicidades, quando comparada à bupivacaína ${ }^{2,3}$. Akerman e col. ${ }^{4}$ demonstraram que a ropivacaína bloqueia mais intensamente as fibras $\mathrm{A} \delta$ e $\mathrm{C}$ (fibras sensitivas), enquanto em outros modelos experimentais em cães o bloqueio motor foi de menor intensidade e duração, comparado ao da bupivacaína ${ }^{5}$.

A anestesia peridural torácica é técnica utilizada com certa freqüência em nosso meio, principalmente em cirurgias plásticas $^{6}$, com a finalidade de reduzir o sangramento no campo operatório. Esta redução é devida à diminuição na pressão arterial. Toxicidade sistêmica por injeção intravascular acidental, injeção subaracnóidea e raquianestesia total, bem como lesões neurológicas são complicações possíveis da técnica. Em recentes publicações ${ }^{7,8}$, foram descritos casos de intoxicação por ropivacaína após bloqueios de nervos periféricos que evoluíram para parada cardíaca prontamente atendida e revertida.

O objetivo destes relatos é apresentar um caso de parada cardíaca e outro de toxicidade neurológica após anestesia peridural torácica realizada com ropivacaína a $0,5 \%$. 


\section{RELATO DOS CASOS}

\section{Caso 1}

Paciente do sexo feminino, 49 anos, $83 \mathrm{~kg}, 170 \mathrm{~cm}$, estado físico ASAII, com antecedentes pessoais de hipertensão arterial em uso irregular de propranolol ( $40 \mathrm{mg} / \mathrm{dia}$ ), suspenso previamente 2 semanas por conta própria, acidente vascular encefálico isquêmico (AVE) sem seqüelas neurológicas, obesidade mórbida e 3 cirurgias para tratamento de hérnias discais lombares, a ser submetida a correção cirúrgica de lipodistrofia de abdômen. Na sala de operação, foi realizada venóclise no membro superior e iniciou-se infusão de solução de Ringer com lactato. Após ter sido instalada monitorização (controle não-invasivo da pressão arterial, oximetria de pulso e eletrocardioscopia na derivação $D_{\|}$) e terem sido administrados $3 \mathrm{mg}$ de midazolam por via venosa, a paciente foi colocada na posição sentada. A punção peridural foi realizada com a técnica de Dogliotti em $\mathrm{T}_{10}-\mathrm{T}_{11}$. Em seguida foi realizada dose-teste com $60 \mathrm{mg}$ de lidocaína a $2 \%(3 \mathrm{ml}) \mathrm{com}$ adrenalina (1:200.000) sem intercorrências e foram administrados $150 \mathrm{mg}$ de ropivacaína a 0,5\% (30 ml). Antes mesmo do término da injeção do anestésico, a paciente referiu zumbido e parestesia perioral, imediatamente seguidos de perda da consciência, convulsões tônico-clônicas, bradicardia, hipotensão arterial e assistolia. Procedeu-se a ventilação com oxigênio sob máscara facial, massagem cardíaca externa e injeção venosa de $1 \mathrm{mg}$ de adrenalina. Seguiu-se a recuperação da função cardíaca, registrando-se valor de pressão arterial de $160 \times 95 \mathrm{mmHg}$, freqüência cardíaca de 125 bpm e oximetria $\left(\mathrm{SpO}_{2}\right)$ em $98 \%$. Novamente a paciente apresentou convulsões, tratadas com tiopental sódico (375 mg), sendo intubada logo após. Foi submetida à anestesia geral com ventilação artificial, utilizando-se alfentanil $\left(30 \mu \mathrm{g} . \mathrm{kg}^{-1}\right)$ e sevoflurano a $1 \%$, controlado pelo analisador de gases marca Dixtal. O bloqueio neuromuscular foi obtido com brometo de rocurônio $\left(0,7 \mathrm{mg} \cdot \mathrm{kg}^{-1}\right)$. Na tentativa de se obter proteção neurológica, foram infundidos dexametasona (10 mg) e manitol a $20 \%\left(1 \mathrm{~g}^{\mathrm{kg}} \mathrm{g}^{-1}\right)$. Como se apresentava hemodinamicamente estável, optou-se pela realização da cirurgia proposta, que durou cerca de duas horas. Ao final do procedimento, foram administrados sulfato de atropina $(1 \mathrm{mg})$ e metilsulfato de neostigmina (2 mg), além de cloridrato de nalbufina (10 mg), para analgesia. A paciente apresentou respiração espontânea e despertar rápidos, sendo extubada ainda na sala de operação. Já na sala de recuperação pós-anestésica (SRPA), não se lembrava de nada, movimentava os membros sem dificuldades e não apresentava dor. Recebeu alta da SRPA duas horas após. Durante acompanhamento por seis meses, após o ocorrido, nenhuma seqüela foi observada.

\section{Caso 2}

Paciente do sexo feminino, 39 anos, $58 \mathrm{~kg}, 165 \mathrm{~cm}$, estado físico ASA I, a ser submetida a cirurgias de implante de próteses mamárias de silicone e lipoaspiração abdominal. Na sala de operação, foi realizada venóclise no membro superior e iniciou-se infusão de solução de Ringer com lactato. Após ter sido instalada monitorização com controle não-invasivo da pressão arterial, oximetria de pulso e eletrocardioscopia na derivação $D_{\|}$e após terem sido administrados 2 mg de midazolam por via venosa, seguidos da infusão contínua de propofol pelo sistema do Diprifusor ${ }^{\circledR}$ (concentração-alvo 1 $\mu \mathrm{g} \cdot \mathrm{ml}^{-1}$ ), a paciente foi colocada na posição sentada. A punção peridural foi realizada pela técnica de Dogliotti em $\mathrm{T}_{8}-\mathrm{T}_{9}$. Procedeu-se, então, à administração de dose-teste com 60 mg de lidocaína a $2 \%$ (3 ml) com adrenalina (1:200.000), sem intercorrências, e $150 \mathrm{mg}$ de ropivacaína a $0,5 \%$. Ao término dos $20 \mathrm{ml}$ (100 mg) inicialmente injetados, na troca das seringas, foi observado refluxo de sangue pela agulha de Tuohy $\left(17 G 3 \frac{1}{2}\right)$, removida imediatamente da paciente que foi rapidamente posicionada em decúbito dorsal. Nesse momento, houve confusão mental, seguida de perda da consciência e convulsões tônico-clônicas. Houve necessidade do aumento da concentração-alvo do propofol para $2,5 \mu \mathrm{g} \cdot \mathrm{ml}^{-1}$ para controle do quadro neurológico e de assistência ventilatória com oxigênio sob máscara facial. A paciente foi intubada e anestesia geral foi realizada com alfentanil $\left(30 \mu \mathrm{g} \cdot \mathrm{kg}^{-1}\right)$ e sevoflurano a $2 \%$, controlado pelo analisador de gases marca Dixtal. A ventilação artificial foi facilitada pela administração de brometo de rocurônio $\left(0,7 \mathrm{mg} \cdot \mathrm{ml}^{-1}\right)$. Em momento algum houve comprometimento do sistema cardiovascular, mantendo-se a pressão arterial em $115 \times 80 \mathrm{mmHg}$, a freqüência cardíaca em 92 bpm e a oximetria $\left(\mathrm{SpO}_{2}\right)$ em $99 \%$, o que permitiu a realização do procedimento cirúrgico proposto. A cirurgia teve a duração de aproximadamente 2 horas e ao término, foram administrados sulfato de atropina ( $1 \mathrm{mg})$ e metilsulfato de neostigmina ( $2 \mathrm{mg}$ ), além de cloridrato de nalbufina (10 mg), para analgesia. A paciente apresentou respiração espontânea e despertar rápidos, sendo extubada na sala de operação. Na SRPA, queixava-se de dor suportável, movimentava os membros sem dificuldades, não se lembrando do ocorrido. Recebeu alta da SRPA 90 minutos após. Durante acompanhamento da paciente, por 6 meses, não foram observadas seqüelas.

\section{DISCUSSÃo}

A decisão de se encontrar outro anestésico local amida, de longa duração de ação, para uso clínico está ligada à história da utilização da bupivacaína nos Estados Unidos. Há vinte e quatro anos, George Albright publicou editorial alertando praticantes da anestesia sobre seis casos quase simultâneos de convulsões e colapsos cardiovasculares após injeção intravascular acidental de etidocaína e bupivacaína ${ }^{1}$. A reanimação foi mal sucedida na maioria desses pacientes. Pesquisas incentivadas por este relatório apresentaram evidências de que a bupivacaína possuía a menor margem terapêutica em relação ao bloqueio do sistema de condução no coração, de acordo com o padrão da época (lidocaína) ${ }^{9-11}$. Ela bloquearia os canais de sódio rapidamente durante o potencial de ação, porém com dissociação mais lenta que a da lidocaína $^{12}$. A depressão da condução elétrica resultante propi- 
ciaria disritmias ventriculares reentrantes. Ainda, publicou-se a sugestão de que elevadas concentrações de bupivacaína poderiam causar disritmias ventriculares por ação direta no tronco encefálico ${ }^{13}$.

A ropivacaína é um anestésico local do grupo amino-amida que difere da bupivacaína por apresentar menor cadeia alifática (propil ao invés de butil) ligada ao nitrogênio do grupo piperidina. Esta substituição, que está associada à menor solubilidade lipídica, e o fato de se apresentar como isômero puro, o enantiômero $S(-)$, conferem à ropivacaína toxicidades neurológica e cardíaca inferiores, em doses iguais, à da mistura racêmica da bupivacaína ${ }^{14,15}$. Em diferentes estudos, concentrações plasmáticas elevadas de ropivacaína, após anestesias regionais (de 2 a $5,6 \mathrm{mg}^{\mathrm{I}^{-1}}$ ), foram alcançadas sem toxicidade neurológica e cardíaca ${ }^{16,17}$. Contudo, vários acidentes após a utilização da ropivacaína têm sido relatados ${ }^{18-25}$. Estes acidentes foram conseqüência de injeções intravasculares diretas (início rápido) ou secundárias à absorção plasmática por excesso de dose (evento clínico tardio).

Mesmo baixas concentrações sangüíneas desse anestésico local podem determinar toxicidade, o que sugere sensibilidade particular dos pacientes a ele, como observado em estudos da toxicidade da ropivacaína realizados por Scotte col. ${ }^{26}$ e Knudsen e col. ${ }^{27}$. Vários voluntários sadios toleraram somente baixas doses e sinais neurológicos ocorreram em concentrações plasmáticas entre 0,5 e $1 \mathrm{mg} . \mathrm{l}^{-1}$, sendo que um paciente tolerou concentração plasmática de $3,2 \mathrm{mg}^{-I^{-1}} 27$. Nos casos apresentados, não foi possível a dosagem da concentração plasmática da ropivacaína.

Knudsen e col. ${ }^{27}$ compararam, ainda, a incidência de sintomas do sistema nervoso central e mudanças na eco e eletrocardiografia durante a infusão de ropivacaína e bupivacaína em voluntários. Reiz e col. ${ }^{2}$ demonstraram, experimentalmente, em porcos, que a proporção da toxicidade eletrofisiológica foi menor para a ropivacaína que para a bupivacaína. A incidência de eventos cardiovasculares e de convulsões induzidos pela ropivacaína é de aproximadamente 6,1 e 8 casos por milhão de pacientes, respectivamente ${ }^{28}$. Em casos relatados, as complicações cardíacas sempre foram precedidas de sinais neurológicos (confusão e convulsões) e, no primeiro caso descrito, isto se confirmou. Estudos recentes ${ }^{15,29}$ confirmam a significativa vantagem, do ponto de vista cardiológico, da ropivacaína sobre a bupivacaína.

A pesquisa experimental revela incidência menor de reanimação cardiopulmonar mal sucedida após ropivacaína, quando comparada à bupivacaína. Em ratos, Ohmura e col. ${ }^{30}$ enfatizaram que a parada cardíaca induzida pela ropivacaína parece ser mais susceptível ao tratamento que aquela provocada pela bupivacaína ou levobupivacaína, o que está de acordo com o que se encontra em seres humanos ${ }^{1,7,8,11}$. Groban e col. ${ }^{31}$ relataram menor incidência de fibrilação ventricular induzida pela epinefrina em cães intoxicados com ropivacaína, quando se comparou com bupivacaína. Além disso, a concentração plasmática no colapso cardiovascular foi maior para a ropivacaína $\left(19,8 \mu \mathrm{g} \cdot \mathrm{ml}^{-1}\right)$, comparada com a bupivacaína $\left(5,7 \mu \mathrm{g} \cdot \mathrm{ml}^{-1}\right)$.
Em voluntários saudáveis, a ropivacaína pode prejudicar a condução e a contração miocárdicas, fato observado somente em concentrações muito mais elevadas que as da bupivacaína, o que sugere melhor tolerância e ratifica resultados em animais ${ }^{26,27}$. Além disso, esses efeitos são mais rapidamente revertidos. Como no caso relatado por Ruetsch e col. $^{32}$, a recuperação cardíaca no caso 1 foi imediata e bem sucedida, não tendo sido observadas disritmias ventriculares. Embora a reanimação cardiopulmonar tenha sido facilitada pelo tratamento precoce, a baixa cardiotoxicidade intrínseca da ropivacaína (quando comparada à da bupivacaína) pode ter sido determinante no resultado. Além disso, o bem sucedido tratamento, que não excedeu um minuto, contrasta com a dificuldade em se reverter quadros de parada cardíaca devidos à intoxicação pela bupivacaína, para os quais se utilizaram até circulação extracorpórea ${ }^{33,34}$, possibilitando que a cirurgia fosse realizada, em concordância com publicações anteriores ${ }^{7,35}$.

Somente a paciente do caso 1 fazia uso, irregularmente, de $\beta$-bloqueador. A presença de antecedentes neurológicos, de episódio de AVE isquêmico sem seqüelas e de três laminectomias descompressivas não interferiram no diagnóstico precoce de intoxicação pela ropivacaína. Como as cirurgias na coluna vertebral foram realizadas nos segmentos lombares $\left(L_{4}-L_{5}\right.$ e $\left.L_{5}-S_{1}\right)$, não havia contra-indicação à punção peridural torácica proposta para a paciente. A realização de anestesia peridural torácica em nosso serviço se faz baseada nas vantagens da técnica ${ }^{36,37}$. A técnica pode ser utilizada como anestesia (cirurgias plásticas), analgesia pós-operatória (toracotomias), dentre outras indicações. A ausência de dor, após o término da anestesia no primeiro caso, leva a supor de que nem todo o anestésico foi injetado no vaso, mas mesmo assim a paciente evoluiu para parada cardiorrespiratória. Já a paciente do caso 2 apresentou comprometimento somente do sistema nervoso, sem alterações do ritmo cardíaco, da $\mathrm{SpO}_{2}$ (\%) ou da pressão arterial, o que já foi observado em estudos publicados a respeito da sensibilidade individual à ropivacaína ${ }^{26,27}$. Quando administrada em infusão venosa a voluntários, Scott e col. ${ }^{26}$ demonstraram o surgimento de convulsões em concentrações plasmáticas de 1 a 2 mg. I $^{-1}$. Já Knudsen e col. ${ }^{27}$ observaram o limiar convulsivo em 2,2 $\mathrm{mg} . \mathrm{I}^{-1}$.

Os casos descritos destacam a possibilidade da injeção intravascular do anestésico local durante a realização da anestesia peridural, mesmo após ter sido administrada a dose-teste. As técnicas utilizadas para detectar injeção intravascular podem reduzir, mas não eliminar, eventos catastróficos. Conseqüentemente, a anestesia regional, utilizando grandes quantidades de anestésicos locais, deve ser realizada em locais com equipamentos para reanimação e por indivíduos treinados para reconhecer estas complicações, para que se inicie precocemente o tratamento ${ }^{38}$.

Foram relatados dois casos de intoxicação por ropivacaína durante a realização da anestesia peridural. No primeiro, a reanimação cardiopulmonar foi bem sucedida e permitiu que a cirurgia fosse realizada, considerando dados prévios que atestam a menor cardiotoxicidade da ropivacaína. No segunVol. 54, N 6, Novembro - Dezembro, 2004 
do, houve comprometimento do sistema nervoso central, não tendo evoluído para toxicidade cardíaca. Não houve seqüelas, traumas, recordações do momento que pudessem comprometer a integridade física e psíquica das pacientes.

\section{Accidental Intravascular Injection of $0.5 \%$ Ropivacaine during Thoracic Epidural Anesthesia. Case Reports}

Fábio Geraldo Curtis, TSA, M.D.; Robson Furlani, M.D.; Yara Marcondes Machado Castiglia, TSA, M.D.

\section{INTRODUCTION}

Ropivacaine has been introduced in the clinical practice as potentially safer than bupivacaine ${ }^{1}$. It is currently widely used in regional anesthesia because of its peculiarities, in particular the lower neuro and cardiotoxicity as compared to bupivacaine ${ }^{2,3}$. Akerman et al. ${ }^{4}$ have shown that bupivacaine produces a more intense block in $A \delta$ and $C$ fibers (sensory fibers), while in other experimental models with dogs, motor block has been less intensive and shorter as compared to bupivacaine ${ }^{5}$.

Thoracic epidural anesthesia is often employed in Brazil, especially for plastic surgeries ${ }^{6}$, since it reduces operative bleeding by decreasing blood pressure. Systemic toxicity due to accidental intravascular injection, spinal injection and total spinal anesthesia, as well as neurological injuries are potential complications of this technique. Recent publications ${ }^{7,8}$ have described ropivacaine-induced intoxication after peripheral nerve block, evolving to cardiac arrest which was promptly treated and reverted.

These reports aimed at presenting a case of cardiac arrest and another one of neurological toxicity after thoracic epidural anesthesia with $0.5 \%$ ropivacaine.

\section{CASE REPORTS}

\section{Case 1}

A 49-year-old female patient, $83 \mathrm{~kg}, 170 \mathrm{~cm}$, physical status ASA II, was scheduled to a abdomen lipodystrophy correction. Her clinical history included arterial hypertension irregularly treated with propranolol (40 mg/day), spontaneously withdrawn 2 weeks before, ischemic encephalic stroke without neurological sequelae, morbid obesity and 3 surgeries to correct herniated lumbar disks.

Venoclysis of upper limb was performed in the operating room and lactated Ringer's solution infusion was started. After monitoring (noninvasive blood pressure, pulse oximetry and $E C G$ at $D_{\|}$lead), patient was given $3 \mathrm{mg}$ intravenous midazolam and then placed in the sitting position. Epidural puncture was performed with the Dogliotty technique at
$\mathrm{T}_{10}-\mathrm{T}_{11}$ space. After a negative test dose with $60 \mathrm{mg}$ of $2 \%$ lidocaine $(3 \mathrm{~mL})$ with epinephrine $(1: 200,000), 150 \mathrm{mg}$ of $0.5 \%$ ropivacaine $(30 \mathrm{~mL})$ were administered and, even before the injection completion, patient referred ear hum and perioral paresthesia, immediately followed by loss of consciousness, tonoclonic seizures, bradycardia, hypotension and asystole.

Patient was ventilated with oxygen under facial mask, external cardiac massage was started and $1 \mathrm{mg}$ intravenous epinephrine was administered, followed by cardiac function recovery with blood pressure $=160 \times 95 \mathrm{mmHg}$, heart rate $=125$ bpm and oximetry $\left(\mathrm{SpO}_{2}\right)=98 \%$. Again patient presented seizures, now treated with sodium thiopental $(375 \mathrm{mg})$, followed by tracheal intubation. Patient was then kept under general anesthesia and mechanical ventilation, with the aid of 30 $\mu \mathrm{g} . \mathrm{kg}^{-1}$ alfentanil and $1 \%$ sevoflurane, monitored by the Dixtal gases analyzer. Neuromuscular block was obtained with $0.7 \mathrm{mg} \cdot \mathrm{kg}^{-1}$ rocuronium.

Aiming at neurological protection, $10 \mathrm{mg}$ dexamethasone and $20 \%$ mannitol $\left(1 \mathrm{~g} \mathrm{~kg}^{-1}\right)$ were infused. Since patient was hemodynamically stable, we decided to proceed with the proposed surgery, which lasted approximately 2 hours. At its completion, $1 \mathrm{mg}$ atropine and $2 \mathrm{mg}$ neostigmine were administered, in addition to $10 \mathrm{mg}$ nalbuphine for postoperative analgesia. Patient had fast spontaneous breathing recovery and emergence, being extubated still in the operating room. In the post-anesthetic recovery unit (PACU), patient could not remember anything, would easily move limbs and did not refer pain, being discharged 2 hours later. No sequelae were observed during 6-month follow up.

\section{Case 2}

A 39-year-old female patient, $58 \mathrm{~kg}, 165 \mathrm{~cm}$, physical status ASA I, was scheduled to mammary silicone prosthesis implant and abdominal liposuction. Venoclysis in upper limb was performed in the operating room and lactated Ringer's solution infusion was started. After monitoring with noninvasive blood pressure, pulse oximetry and ECG at $D_{\|}$ lead, $2 \mathrm{mg}$ intravenous midazolam was administered, followed by continuous propofol infusion by the Diprifusor ${ }^{\circledR}$ system $\left(1 \mu \mathrm{g} \cdot \mathrm{mL}^{-1}\right.$ target-concentration). Patient was then placed in the sitting position.

Epidural puncture was performed with the Dogliotty technique at $T_{8}-T_{9}$. After a negative test-dose of $60 \mathrm{mg}$ of $2 \%$ lidocaine $(3 \mathrm{~mL})$ with epinephrine $(1: 200,000), 150 \mathrm{mg}$ of $0.5 \%$ ropivacaine was administered.

At the end of the initial $20 \mathrm{~mL}(100 \mathrm{mg})$ injection, blood reflux was observed thru the Tuohy needle $\left(17 G_{3} 1 / 2\right)$ when anesthetic syringe was being replaced. The needle was promptly removed and the patient immediately placed in the supine position. At this moment, she presented mental confusion followed by loss of consciousness and tonoclonic seizures. Propofol target concentration had to be increased to 2.5 $\mu \mathrm{g} \cdot \mathrm{mL}^{-1}$ to control neurological symptoms and ventilatory assistance was started with oxygen under facial mask. 
Patient was intubated and general anesthesia was provided by $30 \mu \mathrm{g} \cdot \mathrm{kg}^{-1}$ alfentanil and $2 \%$ sevoflurane, monitored by the Dixtal gases analyzer. Mechanical ventilation was facilitated by $0.7 \mathrm{mg} . \mathrm{kg}^{-1}$ rocuronium. There was no cardiovascular system involvement at any time: blood pressure was maintained at $115 \times 80 \mathrm{mmHg}$, heart rate at $92 \mathrm{bpm}$ and oximetry $\left(\mathrm{SpO}_{2}\right)$ at $99 \%$, what allowed the proposed surgical procedure to be performed. Surgery lasted approximately 2 hours, after what $1 \mathrm{mg}$ atropine and $2 \mathrm{mg}$ neostigmine were administered, in addition to $10 \mathrm{mg}$ nalbuphine for postoperative analgesia. Patient presented fast spontaneous ventilation recovery and emergence, being extubated in the operating room. At PACU she referred bearable pain, could easily move limbs and did not remember the event. Discharged from PACU took place 90 minutes later. No sequelae have been observed during 6 months follow up.

\section{DISCUSSION}

The search for a new long lasting amide local anesthetic for clinical use is linked to the history of bupivacaine in the USA. Twenty-four years ago, George Albright published an editorial warning anesthesia practitioners about 6 almost simultaneous cases of seizures and cardiovascular collapse after accidental intravascular etidocaine and bupivacaine injections ${ }^{1}$. Resuscitation has failed for most those patients. Researches encouraged by this report have shown evidences that bupivacaine had lower therapeutic margin for heart conduction system block, as compared to current standards at that time (lidocaine) ${ }^{9-11}$. Bupivacaine could rapidly block sodium channels during action potential, however with slower dissociation as compared to lidocaine ${ }^{12}$. Resulting electric conduction depression would promote reentering ventricular arrhythmias. There has been also published that high bupivacaine concentrations could promote ventricular arrhythmias by a direct action on the brain stem ${ }^{13}$.

Ropivacaine is an amino-amide local anesthetic differing from bupivacaine for presenting a shorter aliphatic chain (propyl and not butyl) bound to nitrogen of the piperidine group. This replacement, associated to lower lipid solubility, and the fact of being presented as pure isomer - enantiomer $\mathrm{S}(-)$ - provide bupivacaine with lower neurological and cardiac toxicity as compared to racemic bupivacaine in the same dosage ${ }^{14,15}$. In different studies, high ropivacaine plasma concentrations after regional anesthesia ( 2 to $5.6 \mathrm{mg}^{-\mathrm{L}^{-1}}$ ) were reached without neurological and cardiac toxicity ${ }^{16,17}$. However, several ropivacaine-related accidents have been reported ${ }^{18-25}$. These accidents were caused by direct intravascular injections (fast onset) or secondary to plasma absorption due to overdose (late clinical event).

Even low concentrations of this local anesthetic may determine toxicity, suggesting individual sensitivity, as observed in ropivacaine toxicity studies by Scott et al. ${ }^{26}$ and Knudsen et al. ${ }^{27}$. Many healthy volunteers have only tolerated low doses and there were neurological signs with plasma concentrations between 0.5 and $1 \mathrm{mg} . \mathrm{L}^{-1}$. One patient has tolerated 3.2 $\mathrm{mg} \cdot \mathrm{L}^{-1}$ plasma concentration. In our cases, it has not been possible to dose ropivacaine plasma concentrations. Knudsen et al. ${ }^{27}$ have also compared the incidence of central nervous system symptoms and changes in echo and electrocardiography during ropivacaine and bupivacaine infusion in volunteers. Reiz et al. ${ }^{2}$ have experimentally shown in swine that there has been lower electrophysiological toxicity for ropivacaine as compared to bupivacaine. The incidence of ropivacaine-induced cardiovascular events and seizures is of approximately 6.1 and 8 cases per 1 million patients, respectively ${ }^{28}$. In reported cases, cardiac complications were always preceded by neurological signs (confusion and seizures) and, in our first case, this has been confirmed. Recent studies ${ }^{15,29}$ have also confirmed the significant cardiologic advantage of ropivacaine as compared to bupivacaine.

Experimental research has shown lower incidence of failed cardiopulmonary resuscitation after ropivacaine as compared to bupivacaine. In rats, Ohmura et al. ${ }^{30}$ have emphasized that ropivacaine-induced cardiac arrest seems to respond better to treatment than that induced by bupivacaine or levobupivacaine, which is in line with what is seen in humans 1,7,8,11. Groban et al. ${ }^{31}$ have reported lower incidence of epinephrine-induced ventricular fibrillation in dogs intoxicated with ropivacaine as compared to bupivacaine. In addition, plasma concentration during cardiovascular collapse was higher for ropivacaine $\left(19.8 \mu \mathrm{g} \cdot \mathrm{mL}^{-1}\right)$ as compared to bupivacaine $\left(5.7 \mu \mathrm{g} \cdot \mathrm{mL}^{-1}\right)$.

In healthy volunteers, ropivacaine may impair myocardial conduction and contraction, but this is only observed in much higher concentrations than those seen with bupivacaine, suggesting better tolerance and confirming animal results 26,27 . In addition, these effects are more promptly reverted. As in the case reported by Ruetsch et al. ${ }^{32}$, cardiac recovery in case 1 was immediate and successful with no ventricular arrhythmias. Although cardiopulmonary resuscitation was favored by early treatment, intrinsic ropivacaine low toxicity (as compared to bupivacaine) might have determined the outcome. In contrast with the difficult reverting cardiac arrests due to bupivacaine intoxication, where even cardiopulmonary bypass has been used ${ }^{33,34}$, our case's successful treatment - not exceeding one minute - has allowed the surgery to be performed, in line with previous publications ${ }^{7,35}$. Case 1 patient was in irregular use of $\beta$-blockers. The presence of neurological background, ischemic encephalic stroke without sequelae and three decompressive laminectomies has not interfered with early diagnosis of ropivacaine intoxication. Since spinal surgeries were performed in lumbar segments $\left(L_{4}-L_{5}\right.$ and $\left.L_{5}-S_{1}\right)$, there was no counterindication for thoracic epidural puncture proposed for the patient. Thoracic epidural anesthesia in our service is based on the advantages of this technique ${ }^{36,37}$. It may be used for anesthesia (plastic surgeries) or for postoperative analgesia (thoracotomies), among other indications.

The lack of pain after anesthetic recovery in the first case leads to the assumption that not all anesthetic solution was injected into the vessel, but even so, patient has evolved to cardiac arrest. On the other hand, case 2 patient presented only Vol. 54, Nº 6, Novembro - Dezembro, 2004 
nervous system involvement with no cardiac rhythm, $\mathrm{SpO}_{2}$ (\%) or blood pressure changes, which has already been observed in studies on individual sensitivity to ropivacaine ${ }^{26,27}$. When administered in intravenous infusion to volunteers, Scott et al. ${ }^{26}$ have observed seizures with plasma concentrations of 1 to $2 \mathrm{mg} . \mathrm{L}^{-1}$, while Knudsen et al. ${ }^{27}$ have demonstrated a seizure threshold at $2.2 \mathrm{mg} \cdot \mathrm{L}^{-1}$.

This 2 cases report stresses the possibility of intravascular local anesthetic injection during epidural anesthesia, even after test-dose administration. Techniques to detect intravascular injections may decrease, but not rule out catastrophic events. As a consequence, regional anesthesia with high local anesthetic doses should be performed in places where resuscitation equipment is available, and more importantly, by professionals trained to identify these complications and start early treatment ${ }^{38}$

Two cases of ropivacaine intoxication during epidural anesthesia were reported. In the first one, cardiopulmonary resuscitation was successful and allowed for the surgery to be performed, considering previous data attesting lower ropivacaine cardiotoxicity. In the second one, there has been central nervous system involvement not evolving to cardiac toxicity. There have been no sequelae, trauma or remembrances which could have impaired patients' physical and psychic integrity.

\section{REFERÊNCIAS - REFERENCES}

01. Albright GA - Cardiac arrest following regional anesthesia with etidocaine or bupivacaine. Anesthesiology, 1979;51:285-287.

02. Reiz S, Haggmark S, Johansson G et al - Cardiotoxicity of ropivacaine a new amide local anaesthetic agent. Acta Anaesthesiol Scand, 1989;33:93-98.

03. Leisure GS, Di Fasio CA - Ropivacaine: the new local anesthetic. Seminars in Anesthesia, 1996;15:1-9.

04. Akerman B, Hellberg IB, Trossvik C - Primary evaluation of the local anaesthetic properties of the amino amide agent ropivacaine (LEA 103). Acta Anaesthesiol Scand, 1988; 32:571-578.

05. Feldman HS, Covino BG - Comparative motor blocking effects of bupivacaine and ropivacaine, a new amino amide local anesthetic, in the rat and dog. Anesth Analg, 1967;1042-1047.

06. Nociti JR, Serzedo PSM, Cecere A et al - Injeção intravascular acidental de ropivacaina em bloqueio epidural. Relato de caso. Rev Bras Anestesiol, 1999;49:113-114.

07. Chazalon P, Tourtier JP, Villevielle T et al - Ropivacaine-induced cardiac arrest peripheral nerve block: succesful resuscitation. Anesthesiology, 2003;99:1449-1451.

08. Huet O, Eyrolle LJ, Mazoit JX et al - Cardiac arrest after injection of ropivacaine for posterior lumbar plexus blockade. Anesthesiology, 2003;99:1451-1453.

09. Morishima HO, Pedersen H, Finster M et al - Bupivacaine toxicity in pregnant and nonpregnant ewes. Anesthesiology, 1985;63:134-139.

10. Santos AC, Pedersen H, Harmon TW et al - Does pregnancy alter the systemic toxicity of local anesthetics? Anesthesiology, 1989;70:991-995.

11. Morishima HO, Finster M, Arthur GR et al - Pregnancy does not alter lidocaine toxicity. Am J Obstet Gynecol, 1990;162: 1320-1324.
12. Clarkson CW, Hondeghem LM - Mechanism for bupivacaine depression of cardiac conduction: fast block of sodium channels during the action potential with slow recovery from block during diastole. Anesthesiology, 1985;62:396-405.

13. Thomas RD, Behbeham MM, Coyle DE et al - Cardiovascular toxicity of local anesthetics: an alternative hypothesis. Anesth Analg, 1986;65:444-450.

14. Sztark F, Malgat M, Dabadie P et al - Comparison of the effects of bupivacaine and ropivacaine on heart cell mitochondrial bioenergetics. Anesthesiology, 1998;88:1340-1349.

15. Graf BM, Abraham I, Eberbach $N$ et al - Differences in cardiotoxicity of bupivacaine and ropivacaine are the results of physicochemical and stereoselective properties. Anesthesiology, 2002;96:1427-1434.

16. Salonen MH, Haasio J, Bachmann M et al - Evaluation of efficacy and plasma concentrations of ropivacaine in continuous axillary brachial plexus block: High dose for surgical anesthesia and low dose for postoperative analgesia. Reg Anesth Pain Med, 2000;25:47-51.

17. Behnke H, Worthmann F, Cornelissen J et al - Plasma concentration of ropivacaine after intercostal blocks for video-assisted thoracic surgery. Br J Anaesth, 2002;89:251-253.

18. Plowman AN, Bolsin S, Mather LE - Central nervous system toxicity attributable to epidural ropivacaine hydrochloridre. Anaesth Intensive Care, 1998;26:204-206.

19. Bisschop DY, Alardo JP, Razgallah B et al - Seizure induced by ropivacaine. Ann Pharmacother, 2001;35:311-313.

20. Mardirosoff $C$, Dumont $L$ - Convulsions after the administration of high dose ropivacaine following an interescalenic block. Can J Anaesth, 2000;47:1263.

21. Ala-Kokko TI, Lopponen A, Alahuhta S - Two instances of central nervous system toxicity in the same patient following repeated ropivacaine-induced brachial plexus block. Acta Anaesthesiol Scand, 2000;44:623-626.

22. Muller M, Litz RJ, Huler M et al - Grand mal convulsion and plasma concentrations after intravascular injection of ropivacaine for axillary brachial plexus blockade. $\mathrm{Br} \mathrm{J}$ Anaesth, 2001;87:784-187.

23. Ould-Ahmed M, Drouillard I, Fourel D et al - Convulsions induced by ropivacaine after midhumeral block. Ann Fr Anesth Reanim, 2002;21:681-684.

24. Eledjam JJ, Gros T, Viel E et al - Ropivacaine overdose and systemic toxicity. Anaesth Intensive Care, 2000;28:705-707.

25. Petitjeans F, Mion G, Puidupin M et al - Tachycardia and convulsions induced by accidental intravascular ropivacaine injection during sciatic block. Acta Anaesthesiol Scand, 2002;46: 616-617.

26. Scott DB, Lee A, Fagan D et al - Acute toxicity of ropivacaine compared with that of bupivacaine. Anesth Analg, 1989;69: 563-569.

27. Knudsen K, Beckman Suurkula M et al - Central nervous and cardiovascular effects of i.v. infusions of ropivacaine, bupivacaine and placebo in volunteers. $\mathrm{Br} J$ Anaesth, 1997;78:507-514.

28. Periodic Safety Update Report: Naropin, AstraZeneca, May 17, 2000.

29. Ladd LA, Chang DH, Wilson KA et al - Effects of CNS site-directed carotid arterial infusions of bupivacaine, levobupivacaine, and ropivacaine in sheep. Anesthesiology, 2002; $97: 418-428$.

30. Ohmura S, Kawada M, Ohta T et al - Systemic toxicity and resuscitation in bupivacaine, levobupivacaine, or ropivacaine-infused rats. Anesth Analg, 2001:93:743-748. 
31. Groban L, Deal DD, Vernon JC et al - Cardiac resuscitation after incremental overdosage with lidocaine, bupivacaine, levobupivacaine, and ropivacaine in anesthetized dogs. Anesth Analg, 2001;92:37-43.

32. Ruetsch YA, Fattinger KE, Borgeat A - Ropivacaine-induced convulsions and severe cardiac dysrhythmia after sciatic block. Anesthesiology, 1999;90:1784-1786.

33. Long WB, Rosenblum S, Grady IP - Successful resuscitation of bupivacaine-induced cardiac arrest using cardiopulmonary bypass. Anesth Analg, 1989;69:403-406.

34. Tsai MH, Tseng CK, Wong KC - Successful resuscitation of a bupivacaine-induced cardiac arrest using cardiopulmonary bypass and mitral valve replacement. J Cardiothorac Anesth, 1987; 1:454-456.

35. Abouleish El, Elias M, Nelson C - Ropivacaine-induced seizure after extradural anaesthesia. Br J Anaesth, 1998;80:843-844.

36. Nociti JR, Serzedo PS, Zuccolotto EB et al - Ropivacaína em bloqueio peridural torácico para cirurgia plástica. Rev Bras Anestesiol, 2002;52:156-165.

37. Leão DG - Peridural torácica: estudo retrospectivo de 1240 casos. Rev Bras Anestesiol, 1997;47:138-147.

38. Klein SM, Pierce T, Rubin Y et al - Successful resuscitation after ropivacaine-induced ventricular fibrillation. Anesth Analg, 2003;97:901-903.

\section{RESUMEN}

Curtis FG, Furlani R, Castiglia YMM - Inyección Intravascular Accidental de Ropivacaína a 0,5\% durante la Realización de Anestesia Peridural Torácica. Relato de Casos

JUSTIFICATIVA Y OBJETIVOS: A ropivacaína fue introducida en la práctica clínica hace poco más de diez años, asociándose a bajo riesgo de complicaciones del sistema nervioso central y cardiovascular. Estos relatos tienen como objetivo presentar un caso de parada cardíaca y otro de toxicidad neurológica, después de inyección intravascular accidental de ropivacaína, durante la realización de anestesias peridurales.

RELATO DE LOS CASOS: Se trata de dos pacientes sometidas a cirugías plásticas estéticas bajo anestesia peridural torácica con ropivacaína a $0,5 \%$. Durante la realización de la técnica, una de ellas presentó parada cardíaca en asistolia y la otra, toxicidad neurológica. Prontamente atendidas, ambas presentaron rápida recuperación, habiendo sido posible la realización de los respectivos actos quirúrgicos.

CONCLUSIONES: EI reconocimiento y el tratamiento rápidos de la inyección intravascular accidental, bien como las características farmacológicas de la ropivacaína fueron decisivos, en ambos casos, con una buena recuperación de las pacientes. 